\title{
Spin Hall effect in a spin-1 chiral semimetal
}

\author{
Ke Tang $\odot,{ }^{1,2}$ Yong-Chang Lau $\odot,{ }^{3,4}$ Kenji Nawa $\odot,{ }^{1,5}$ Zhenchao Wen $\odot,{ }^{1, *}$ Qingyi Xiang, ${ }^{1}$ Hiroaki Sukegawa $\odot,{ }^{1}$ \\ Takeshi Seki, ${ }^{3,4}$ Yoshio Miura, ${ }^{1}$ Koki Takanashi, ${ }^{3,4,6}$ and Seiji Mitani ${ }^{1,2}$ \\ ${ }^{1}$ National Institute for Materials Science (NIMS), Tsukuba 305-0047, Japan \\ ${ }^{2}$ Graduate School of Pure and Applied Sciences, University of Tsukuba, Tsukuba 305-8577, Japan \\ ${ }^{3}$ Institute for Materials Research, Tohoku University, Sendai 980-8577, Japan \\ ${ }^{4}$ Center for Spintronics Research Network, Tohoku University, Sendai 980-8577, Japan \\ ${ }^{5}$ Graduate School of Engineering, Mie University, Tsu 514-8507, Japan \\ ${ }^{6}$ Center for Science and Innovation in Spintronics, Core Research Cluster, Tohoku University, Sendai 980-8577, Japan
}

(Received 7 April 2021; revised 24 June 2021; accepted 28 June 2021; published 29 July 2021)

\begin{abstract}
The spin- 1 chiral semimetal is a state of quantum matter hosting unconventional chiral fermions that extend beyond the common Dirac and Weyl fermions. B20-type CoSi is a prototypal material that accommodates such an exotic quasiparticle. To date, the spin-transport properties in the spin-1 chiral semimetals have not been thoroughly explored. In this work, we fabricated $B 20-\mathrm{CoSi}$ thin films on sapphire $c$-plane substrates by magnetron sputtering and studied the spin Hall effect (SHE) by combining experiments and first-principles calculations. The SHE of $\mathrm{CoSi}$ was investigated using $\mathrm{CoSi} / \mathrm{CoFeB} / \mathrm{MgO}$ heterostructures via spin Hall magnetoresistance and harmonic Hall measurements. First-principles calculations yield an intrinsic spin Hall conductivity (SHC) at the Fermi level that is consistent with the experiments and reveal its unique Fermi-energy dependence. Unlike the Dirac and Weyl fermion-mediated Hall conductivities that exhibit a peaklike structure centering around the topological node, SHC of $B 20-\mathrm{CoSi}$ is odd and crosses zero at the node with two antisymmetric local extrema of opposite sign situated below and above in energy. Hybridization between Co $d$-Si $p$ orbitals and spin-orbit coupling are essential for the SHC, despite the small $(\sim 1 \%)$ weight of the Si $p$ orbital near the Fermi level. This work expands the horizon of topological spintronics and highlights the importance of Fermi-level tuning in order to fully exploit the topology of spin-1 chiral fermions for spin-current generation.
\end{abstract}

DOI: 10.1103/PhysRevResearch.3.033101

\section{INTRODUCTION}

Spin current generated from the spin Hall effect (SHE) can exert spin-orbit torque (SOT) on an adjacent ferromagnetic (FM) nanolayer, which provides a promising way to manipulate the magnetization of the FM layer for high-performance spintronic devices [1,2], such as SOT magnetoresistive random access memories (MRAMs). For applications involving SOT-based devices, it is important to find materials exhibiting large dampinglike spin Hall efficiency $\left(\xi_{\text {DL }}\right)$, i.e., the efficiency of spin-charge conversion of SHE. Heavy $5 d$ transition metals, such as $\mathrm{Pt}, \mathrm{Ta}$, and $\mathrm{W}$, are commonly known for possessing large $\xi_{\mathrm{DL}}$ due to their strong spin-orbit interactions and optimum $d$-orbital filling [3-6]. Topological insulators (TIs), such as $\mathrm{Bi}_{2} \mathrm{Se}_{3}$ [7-10], were reported to show even larger $\xi_{\mathrm{DL}}$, which arises from the spin-momentum locking of their surface states. However, several complications hinder the integration of the TIs to the state-of-the-art electronics: First,

\footnotetext{
*wen.zhenchao@nims.go.jp

Published by the American Physical Society under the terms of the Creative Commons Attribution 4.0 International license. Further distribution of this work must maintain attribution to the author(s) and the published article's title, journal citation, and DOI.
}

the relatively low melting point of these Bi-based TIs is not compatible with the complementary metal oxide semiconductor processing. Second, the bulk-insulating TIs are generally too resistive to be incorporated into devices such as SOT MRAMs. Third, in a multilayer heterostructure, the surface states may be altered when a TI is in direct contact with a ferromagnet.

The emergence of topological Weyl semimetals (WSMs) provides a promising alternative for efficient spin-current generation in SOT devices, due to the topological nature of the bulk band structures and the relatively low resistivity of WSMs compared to TIs. The WSMs hold three-dimensional linearly dispersive band-crossing points, where the degeneracy is lifted by breaking either the inversion symmetry or the time-reversal symmetry or both [11]. First-principles calculations have predicted a large intrinsic SHE peak near the Weyl points for the TaAs family of WSMs, where the SHE is interpreted as the interplay of the large spin Berry curvature and spin-orbit coupling (SOC) near the Weyl nodes [12]. Recently, large SHE was observed in Weyl semimetal prototypes $\mathrm{WTe}_{2}$ $[13,14]$ and $\mathrm{Co}_{2} \mathrm{MnGa}[15,16]$.

The cobalt monosilicide CoSi that crystallizes in the $B 20$ structure is a newly discovered topological semimetal featuring unconventional chiral fermions with no counterparts in high-energy physics [17]. The left panel of Fig. 1(a) shows an illustration of the unit cell of cubic B20-structured CoSi. 
(a)

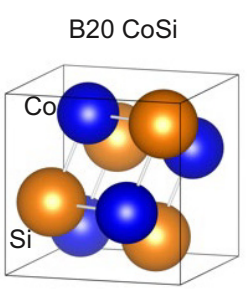

(c)

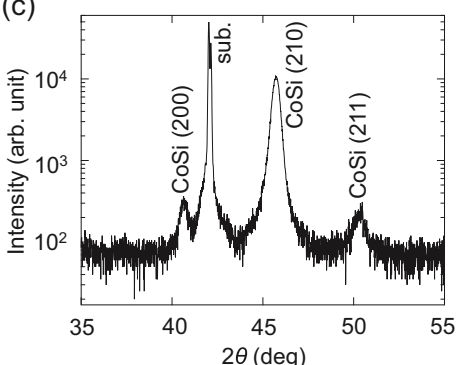

(b) RHEED pattern of CoSi film

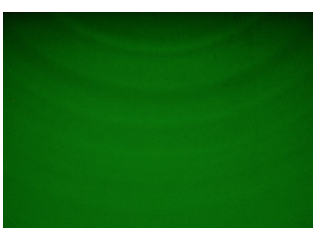

(d)

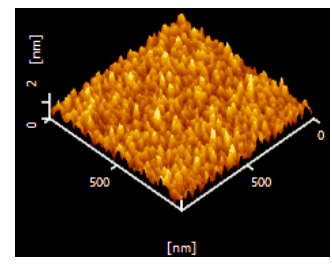

FIG. 1. Structural properties of CoSi films and heterostructure for SOT devices. (a) Unit cell of the cubic B20 crystal structure of $\mathrm{CoSi}$ and schematic of the band structures of a spin- 1 fermion. (b) RHEED pattern of CoSi $(47 \mathrm{~nm})$ film surface deposited on a sapphire $c$-plane substrate. (c) The out-of-plane XRD spectrum of substrate/CoSi(47)/ $\mathrm{Mg}_{2} \mathrm{AlO}_{x}(2 \mathrm{~nm})$. (d) The surface morphology of multilayer stacks with the structure of $\mathrm{CoSi} / \mathrm{CoFeB} / \mathrm{MgO}$ for SOT devices.

The spin-1 chiral fermion holds a threefold degeneracy at the band-crossing node of a Dirac-like band and a flat band, which carries a topological charge of $\pm 2[17,18]$, as illustrated in the right panel of Fig. 1(a). The presence of spin-1 chiral fermions [schematic shown in the right panel of Fig. 1(a)] at the band-crossing points near the Fermi level with long Fermi arcs in the momentum space of CoSi was confirmed by angle-resolved photoemission spectroscopy experiments [19-21]. Fabrication of high-quality CoSi thin films and understanding the spin transport in these topological semimetal thin films are essential steps towards realizing novel spintronic devices exploiting the unique properties of unconventional chiral fermions. To date, there have been few reports on the spin-transport properties of nanometer-scale CoSi thin films and it is not clear yet how the spin-1 chiral electronic structure participates in the spin transport, e.g., spin-current generation via SHE.

In this work, we fabricated CoSi thin films by magnetron sputtering and investigated the spin-transport properties by experiments and first-principles calculations. Structural characterization indicates that the CoSi films are polycrystalline and crystallize in the B20-type structure. Spin Hall magnetoresistance (SMR) and harmonic Hall measurements show that the $\xi_{\mathrm{DL}}$ of $\mathrm{CoSi}$ is $\sim 3 \%$, which is larger than the values reported in pure $\mathrm{Co}(\sim 1 \%)$ [22] and $\mathrm{Si}(\sim 0.01 \%)$ [23]. The first-principles calculations show that the amplitude of the spin Berry curvature depends on the $k$-point path and changes sign above and below the topological node hosting the spin-1 chiral fermion, in contrast to a WSM. The spin Berry curvature contributes to the spin Hall conductivity (SHC) in the $\mathrm{CoSi}$ thin films and originates from the hybridization of $d-p$ orbitals between $\mathrm{Co}$ and $\mathrm{Si}$.

\section{EXPERIMENTAL SECTION}

\section{A. Film characterizations}

CoSi thin films were deposited on sapphire $\left(\mathrm{Al}_{2} \mathrm{O}_{3}\right) c$ plane substrates from a sintered $\mathrm{CoSi}$ alloy target. The composition of the CoSi films was confirmed to be 50.7:49.3 using an inductively coupled plasma optical emission spectrometer (ICP OES). The surface structure of the CoSi films was monitored by in situ reflection high energy electron diffraction (RHEED) in the sputtering chamber. Figure 1(b) is a typical RHEED pattern for a 47-nm-thick CoSi film deposited at $550{ }^{\circ} \mathrm{C}$. The RHEED pattern shows arc-shape diffractions, indicating a polycrystalline surface. The crystal structure was further characterized by x-ray diffraction (XRD) with $\mathrm{Cu} K \alpha$ radiation and a monochromator. Figure 1(c) shows the XRD spectrum of substrate $/ \mathrm{CoSi}(47) / \mathrm{Mg}_{2} \mathrm{Al}-\mathrm{O}_{x}$ (2) (thicknesses in nanometers), where $\mathrm{Mg}_{2} \mathrm{Al}-\mathrm{O}_{x}$ serves as a capping layer to protect the CoSi surface from oxidization. In addition to a diffraction peak from the sapphire substrate, the peaks corresponding to the (200), (210), and (211) crystal planes of B20-type CoSi can be clearly identified, which indicates the polycrystalline nature of the CoSi film without observable secondary phases. From the intensities of the diffraction peaks, it is also known that the CoSi film has a (210)-preferential texture. The lattice constant of the CoSi is estimated to be $\sim 0.4435 \mathrm{~nm}$. Next, a heterostructure consisting of substrate/ $\mathrm{CoSi}\left(t_{\mathrm{CoSi}}: 0-11\right) / \mathrm{Co}_{20} \mathrm{Fe}_{60} \mathrm{~B}_{20}(\mathrm{CoFeB})$ (1)/ $\mathrm{MgO}(2) / \mathrm{Ta}$ (1) was deposited in order to evaluate the spintransport properties of the CoSi film. The surface morphology of the stack layers was measured by atomic force microscopy (AFM). As shown in Fig. 1(d), a relatively flat surface with an average roughness $\left(R_{\mathrm{a}}\right) \sim 0.39 \mathrm{~nm}$ and a peak-to-valley $(P-V) \sim 3.92 \mathrm{~nm}$ for a $1 \times 1 \mu \mathrm{m}^{2}$ scan was achieved. The heterostructure with such a low roughness is essential for spin-transport measurements, which will be further elaborated in the following sections.

\section{B. Spin Hall magnetoresistance measurements}

The multilayer stacks with the core structure of $\mathrm{CoSi} / \mathrm{CoFeB} / \mathrm{MgO}$ were patterned into Hall bars, as shown in Fig. 2(a). SMR was measured to evaluate $\xi_{\text {DL }}$ of the $\mathrm{CoSi}$ thin films. In nonmagnetic/ferromagnetic (NM/FM) layered structures, when a charge current is applied, a spin current can be generated by SHE in the NM and it flows towards the FM. At the interface of the NM/FM, a part of the spin current is reflected back and it is converted to a charge current within the NM by the inverse SHE, resulting in a change of the longitudinal resistance $\left(R_{x x}\right)$ in the heterostructure. The difference in the $R_{x x}$ between parallel and perpendicular configurations of the FM layer magnetization $(\boldsymbol{M})$ and the spin-current polarization $(\boldsymbol{\sigma} / / \boldsymbol{y})$ is referred to as the SMR [24,25]. Neglecting the imaginary part of the spin mixing conductance, $\xi_{\mathrm{DL}}$ of the NM layer can be obtained from the NM layer thickness dependence of the SMR.

Figure 2(a) illustrates the experimental setup for the SMR measurement and the microscope image of a Hall bar. A magnetic field of $20 \mathrm{kOe}$ was applied to saturate the magnetization of the ferromagnetic $\mathrm{CoFeB}$ layer. By rotating the magnetic field in the $z y$ plane, the parallel and perpendicular 
(a)
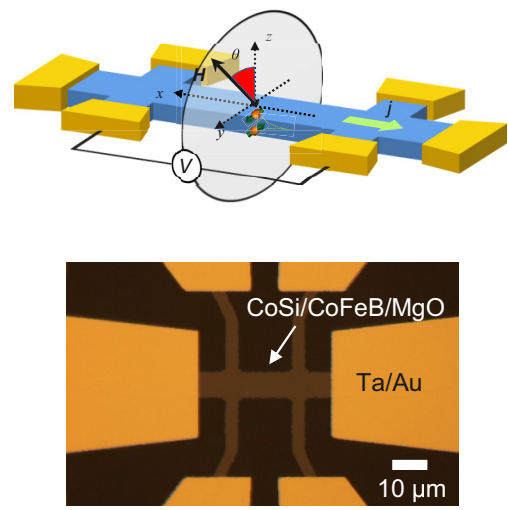

(c)

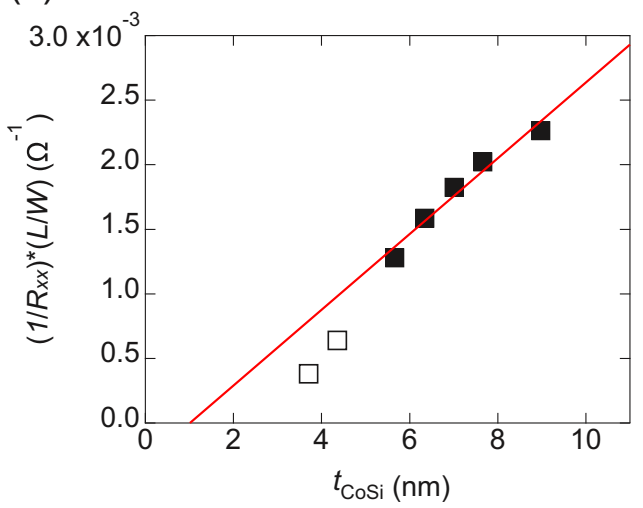

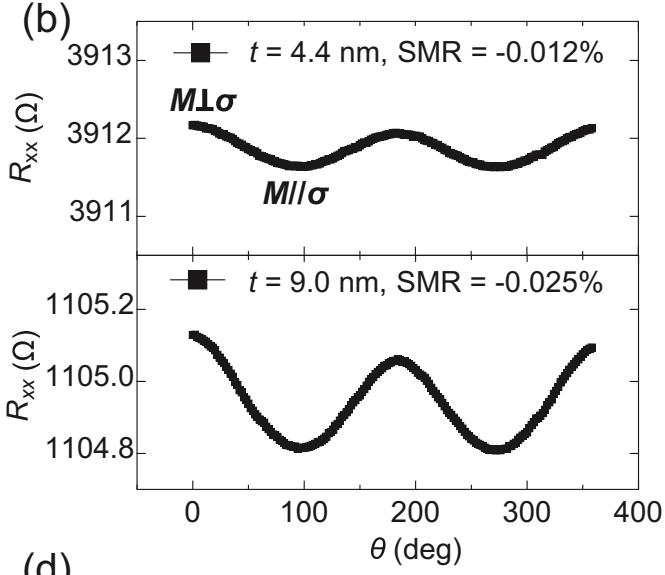

(d)

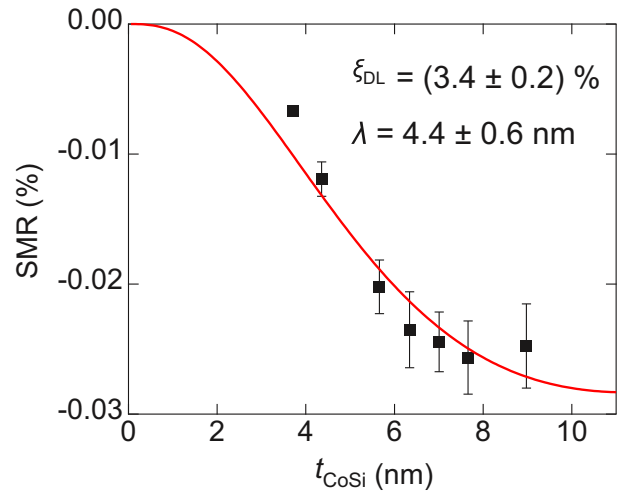

FIG. 2. The measurement of SMR in CoSi. (a) Illustration of a Hall bar device with the measurement setup and coordinate system, and an optical microscope image of a Hall bar device. (b) Plots of the dependence of resistance on the angle between $M$ and $\sigma$ for two Hall bar devices with $t_{\mathrm{CoSi}}=4.4$ and $9.0 \mathrm{~nm}$. (c) Inverse of the sheet resistance as a function of CoSi thickness. The red solid line represents the linear fitting curve in a proper range shown by the black solid squares. (d) Extracted SMR ratio as a function of CoSi thickness. Red solid line is the fitting curve using Eq. (1).

configurations of $\boldsymbol{M}$ and $\boldsymbol{\sigma}$ were achieved. Figure 2(b) shows the $R_{x x}$ change with the orientation of $\boldsymbol{M}$ for two Hall bar devices with $t_{\mathrm{CoSi}}=4.4 \mathrm{~nm}$ and $9.0 \mathrm{~nm}$. The $\vartheta=0^{\circ}$ and $90^{\circ}$ correspond to the perpendicular and parallel states between $\boldsymbol{M}$ and $\boldsymbol{\sigma}$, respectively. The SMR is derived by the ratio of the $R_{x x}$ change, which is given by $\left(R_{x x, \vartheta=90^{\circ}}-R_{x x, \vartheta=0^{\circ}}\right) / R_{x x, \vartheta=0^{\circ}}$. Because the $\boldsymbol{M}$ is always perpendicular to the charge current, there is no contribution of anisotropic magnetoresistance (AMR) to the resistance change. Assuming a transparent interface between $\mathrm{CoSi}$ and $\mathrm{CoFeB}$, the SMR as a function of $t_{\mathrm{CoSi}}$ can be fitted by the following equation derived from a drift-diffusion model [25-27]:

$$
\mathrm{SMR}=-\xi_{\mathrm{DL}}^{2} \frac{\lambda}{t_{\mathrm{CoSi}}} \frac{\tanh \left(t_{\mathrm{CoSi}} / 2 \lambda\right)}{1+\alpha}\left[1-\frac{1}{\cosh \left(t_{\mathrm{CoSi}} / \lambda\right)}\right]
$$

The $\xi_{\mathrm{DL}}$ and the spin diffusion length $(\lambda)$ are used as fitting parameters. The $\alpha$ is given by $\rho_{\mathrm{CoSi}} t_{\mathrm{CoFeB}} / \rho_{\mathrm{CoFeB}} t_{\mathrm{CoSi}}$, where $\rho_{\mathrm{CoSi}}, \rho_{\mathrm{CoFeB}}$, and $t_{\mathrm{CoFeB}}$ represent the resistivity of $\mathrm{CoSi}$, and the resistivity and the thickness of $\mathrm{CoFeB}$, respectively. Here, $t_{\mathrm{CoFeB}}$ is $1 \mathrm{~nm}$ and $\rho_{\mathrm{CoFeB}}$ is $200 \mu \Omega \mathrm{cm}$. The value of $\rho_{\mathrm{CoSi}}$ is derived by fitting the inverse of the sheet resistance of the film as a function of $t_{\mathrm{CoSi}}$, as shown in Fig. 2(c). The inverse of the sheet resistance is defined as $\left(1 / R_{x x}\right) \times(L / W)$, where $L=25 \mu \mathrm{m}$ is the length and $W=10 \mu \mathrm{m}$ is the width of the Hall bar. Thus, the slope of the linear fitting curve represents the inverse of the $\rho_{\mathrm{CoSi}}$. We fitted the data in an appropriate range and estimated that the $\rho_{\mathrm{CoSi}}$ is around 341 $\mu \Omega \mathrm{cm}$. The value of $\rho_{\mathrm{CoSi}}$ is relatively large compared to the reported resistivity of a bulk CoSi single crystal [28], which is attributed to the carrier scattering at the surfaces and the grain boundaries of the polycrystalline thin film. After extracting the SMR of bilayers with different thicknesses of CoSi, we fitted the SMR as a function of $t_{\mathrm{CoSi}}$ by Eq. (1). The experimental data are well fitted, as shown in Fig. 2(d). The $\xi_{\mathrm{DL}}$ and $\lambda$ are obtained to be $3.4 \pm 0.2 \%$ and $4.4 \pm 0.6 \mathrm{~nm}$, respectively. The value of $\xi_{\mathrm{DL}}$ is relatively large even for a material consisting of relatively light elements. Compared to pure $\mathrm{Co}\left(\xi_{\mathrm{DL}} \sim 1 \%\right)$ [22] and $\mathrm{Si}\left(\xi_{\mathrm{DL}} \sim 0.01 \%\right)$ [23], the result indicates that the orbital hybridization between Co and Si orbitals may play a significant role on the SHE in the CoSi, which is supported by the first-principles calculations discussed later.

\section{Harmonic Hall measurements}

The efficiency of spin-current generation from the CoSi thin films was further characterized by harmonic Hall measurements. The SOTs induced by spin current acting on a FM layer can be considered, in the quasistatic regime, as effective magnetic fields, which consist of a dampinglike $\left(\boldsymbol{H}_{\mathrm{DL}} \| \boldsymbol{M} \times \boldsymbol{\sigma}\right)$ term and a fieldlike $\left(\boldsymbol{H}_{\mathrm{FL}} \|-\boldsymbol{\sigma}\right)$ term [29-34]. 
(a)
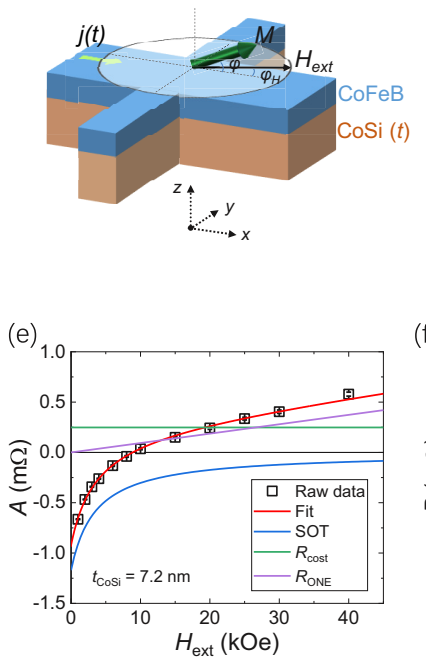

(b)

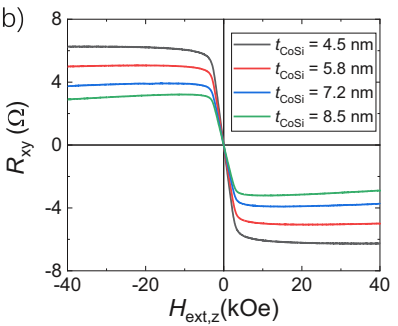

(f) 0.15

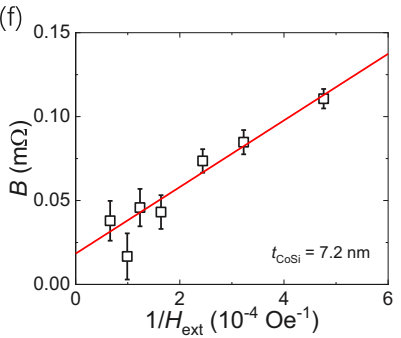

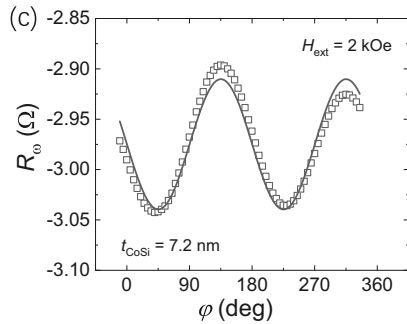

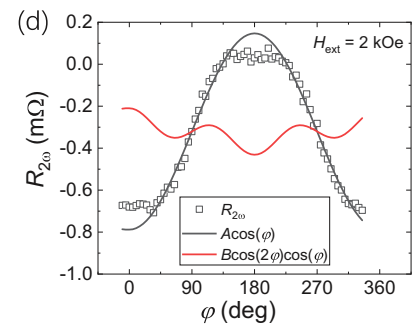

(g)

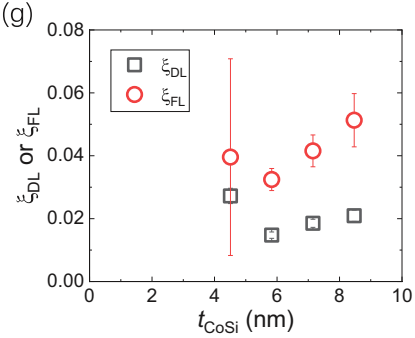

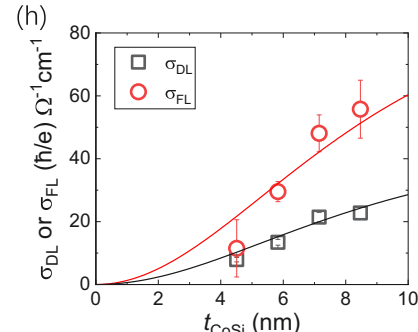

FIG. 3. Harmonic Hall measurement at room temperature for quantifying the SOTs generated from CoSi films. (a) Schematic of the setup for the harmonic Hall measurement. (b) Hall resistance measured under an out-of-plane magnetic field for Hall bar devices with $t_{\mathrm{CoSi}}=4.5$, 5.8, 7.2, and $8.5 \mathrm{~nm}$. (c) First-harmonic Hall resistance $R_{\omega}$ as a function of $\varphi$. Solid line is the fitted curve by Eq. (2). (d) Second-harmonic Hall resistance $R_{2 \omega}$ as a function of $\varphi$. Solid curves are the decomposed components of $R_{2 \omega}$ based on Eq. (3). $R_{\omega}$ and $R_{2 \omega}$ shown in (c,d) were measured at $H_{\mathrm{ext}}=2 \mathrm{kOe}$ for the device with $t_{\mathrm{CoSi}}=7.2 \mathrm{~nm}$. (e) Parameters $A$ and (f) $B$ as a function of $H_{\mathrm{ext}}$ and the inverse of $H_{\mathrm{ext}}$, respectively. (g) CoSi thickness dependence of $\xi_{\mathrm{DL}}$ and $\xi_{\mathrm{FL}}$. (h) $\sigma_{\mathrm{SH}}$ and $\sigma_{\mathrm{FL}}$ as a function of CoSi thickness. The solid lines are the fitting curves by Eq. (5).

The effective fields modulate the orientation of $\boldsymbol{M}$ of the FM layer, resulting in a second-harmonic Hall resistance. The $\xi_{\mathrm{DL}}$ and the fieldlike spin Hall efficiency $\left(\xi_{\mathrm{FL}}\right)$ can be extracted from the azimuthal magnetic field angular dependence of the first- $\left(R_{\omega}\right)$ and the second- $\left(R_{2 \omega}\right)$ harmonic Hall resistances. A schematic illustration of the harmonic Hall measurement is shown in Fig. 3(a). A sinusoidal charge current was applied along the $x$ axis, and an external magnetic field $\boldsymbol{H}_{\text {ext }}$ was applied and rotated in the $x y$ plane, making an angle $\varphi$ with the current. The $R_{\omega}$ and $R_{2 \omega}$ were measured as a function of $\varphi$ at various $H_{\text {ext }}$. The dependences of the first- and the secondharmonic Hall resistances on the angle $\varphi$ can be expressed by the following equations [29],

$$
\begin{aligned}
& R_{\omega}=R_{\mathrm{AHE}} \cos \theta+R_{\mathrm{PHE}} \sin 2 \varphi \sin ^{2} \theta, \\
R_{2 \omega}= & -\left(R_{\mathrm{AHE}} \frac{H_{\mathrm{DL}}}{H_{\mathrm{ext}}+H_{\mathrm{k}}}+R_{\mathrm{cost}}+R_{\mathrm{ONE}}\right) \cos \varphi \\
& +\left(2 R_{\mathrm{PHE}} \frac{H_{\mathrm{FL}}+H_{\mathrm{Oe}}}{H_{\mathrm{ext}}}\right) \cos 2 \varphi \cos \varphi \\
= & A \cos \varphi+B \cos 2 \varphi \cos \varphi,
\end{aligned}
$$

where $R_{\mathrm{AHE}}, R_{\mathrm{PHE}}, H_{\mathrm{k}}$, and $H_{\mathrm{Oe}}$ are the anomalous Hall resistance, the planar Hall resistance, the out-of-plane magnetic anisotropy field of $\mathrm{CoFeB}$, and the Oersted field. $R_{\text {cost }}$ and $R_{\mathrm{ONE}}$ describe $R_{2 \omega}$ contributions of thermoelectric origin that are constant in field (e.g., due to the anomalous Nernst effect of $\mathrm{CoFeB}$ ) and linear in field [e.g., due to the ordinary Nernst effect (ONE) of CoSi], respectively. The $R_{2 \omega}$ is decomposed into two components, i.e., $A \cos \varphi$ and $B \cos 2 \varphi \cos \varphi$.

Figure $3(\mathrm{~b})$ shows the dc Hall resistance $\left(R_{x y}\right)$ of Hall devices with $t_{\mathrm{CoSi}}=4.5,5.8,7.2$, and $8.5 \mathrm{~nm}$. The Hall resistance was measured under an out-of-plane external field
$\left(H_{\mathrm{ext}, z}\right)$ sweeping between -40 and $40 \mathrm{kOe}$. The values of both $R_{\mathrm{AHE}}$ and $H_{\mathrm{k}}$ are estimated from the plot of $R_{x y}$ against $H_{\mathrm{ext}, z}$. The CoFeB layer is in-plane magnetized as shown by the $R_{x y}$ against $H_{\mathrm{ext}, z}$ in Fig. 3(b). Linear fits were performed to the high-field data to eliminate the ordinary Hall effect. $R_{\mathrm{AHE}}$ is obtained from the intercept of the fitted curve. $H_{\mathrm{k}}$ is determined by the magnetic field for saturating $R_{x y}$ after subtracting the contribution of the ordinary Hall effect. The harmonic Hall measurement was carried out by applying a current density of the order of $j_{\mathrm{CoSi}} \sim 1.6 \times 10^{6} \mathrm{~A} / \mathrm{cm}^{2}$ in CoSi. Figure 3(c) shows the $\varphi$ dependence of $R_{\omega}$ for a Hall device with $t_{\mathrm{CoSi}}=7.2 \mathrm{~nm}$ at $H_{\mathrm{ext}}=2 \mathrm{kOe}$. Since $\boldsymbol{M}$ lies in the $x y$ plane $\left(\vartheta=90^{\circ}\right)$, the $R_{\omega}-\varphi$ curve is well fitted by Eq. (2). The $R_{\mathrm{PHE}}$ is obtained to be $65 \mathrm{~m} \Omega$. $R_{2 \omega}$ as a function of $\varphi$ for the same device is plotted in Fig. 3(d). The $A \cos \varphi$ and $B \cos 2 \varphi \cos \varphi$ components of $R_{2 \omega}$ are extracted by fitting the experimental data using Eq. (3). The factor $A$ as a function of $H_{\text {ext }}$ for the device with $t_{\mathrm{CoSi}}=7.2 \mathrm{~nm}$ is plotted in Fig. 3(e). The best fit using Eq. (3) is shown by the red line and other colored lines represent decomposition of the signal. The SOT signal dominates in the low-field regime whereas the $H_{\text {ext }}$ dependence of $R_{2 \omega}$ at higher fields is governed by the ONE. We note that a previous report [28] found appreciable ONE in CoSi single crystal at lower temperatures. The $H_{\mathrm{DL}}$ is estimated to be 1.00 Oe. Figure 3(f) shows the dependence of the factor $B$ against the inverse of $H_{\text {ext }}$. Combining with the $R_{\mathrm{PHE}}$ obtained above and the $H_{\mathrm{Oe}}$ evaluated by the Ampère's law, the $H_{\mathrm{FL}}$ opposing the Oersted field is estimated to be 2.25 Oe from the slope of the linear curve fit in Fig. 3(f). The $\xi_{\mathrm{DL}}$ and $\xi_{\mathrm{FL}}$ are given by [35]

$$
\xi_{\mathrm{DL}(\mathrm{FL})} \equiv \frac{2 e}{\hbar} \frac{H_{\mathrm{DL}(\mathrm{FL})} M_{\mathrm{s}} t_{\mathrm{CoFeB}}}{j_{\mathrm{CoSi}}},
$$


(a)

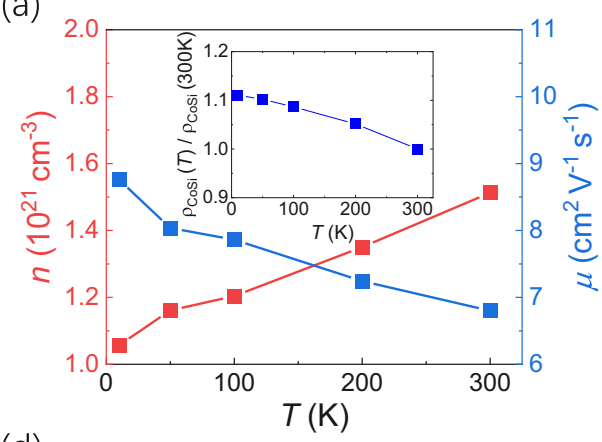

(d)

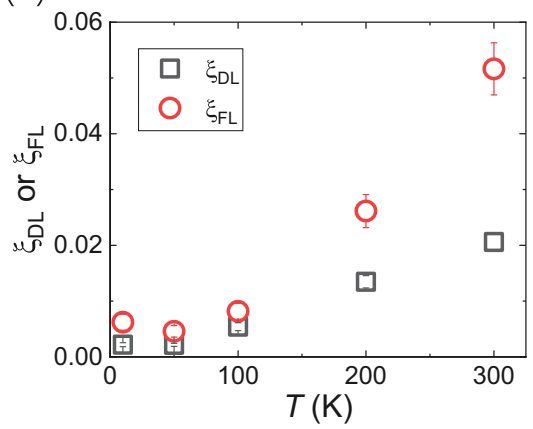

(b)

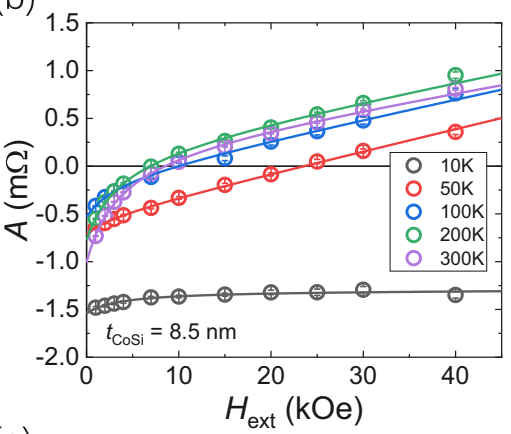

(e)

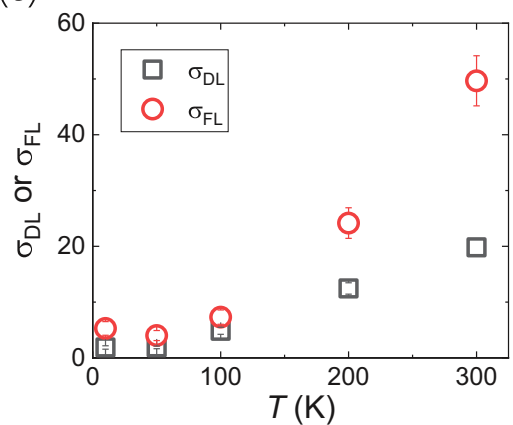

(c)

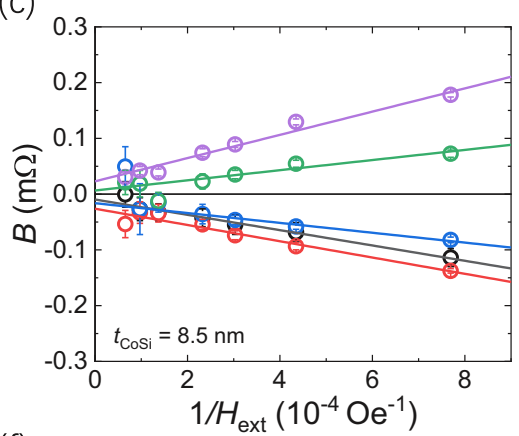

(f)

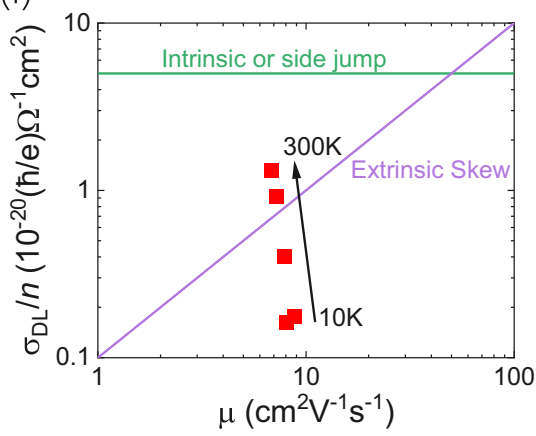

FIG. 4. Temperature dependence of transport properties and SOTs for CoSi films and CoSi/CoFeB heterostructures. (a) The dependence of carrier concentration, mobility, and normalized resistivity on temperature. (b) Parameters $A$ and (c) $B$ as a function of $H_{\text {ext }}$ and the inverse of $H_{\text {ext }}$, respectively, at different temperatures. (d) Temperature dependences of $\xi_{\mathrm{DL}}\left(\xi_{\mathrm{FL}}\right)$ and (e) $\sigma_{\mathrm{DL}}\left(\sigma_{\mathrm{FL}}\right)$. (f) $\sigma_{\mathrm{DL}} / n$ as a function of the carrier mobility for the $\mathrm{CoSi} / \mathrm{CoFeB}$ heterostructures.

where $e$ is the elementary charge, $\hbar$ is the reduced Plank constant, and $M_{\mathrm{s}}=980 \mathrm{emu} / \mathrm{cm}^{3}$ is the saturation magnetization of $\mathrm{CoFeB}$. The CoSi thickness dependences of both $\xi_{\mathrm{DL}}$ and $\xi_{\mathrm{FL}}$ are shown in Fig. 3(g). The result is comparable to the spin Hall efficiency estimated by SMR measurement. The increase in the $\xi_{\mathrm{DL}}$ and $\xi_{\mathrm{FL}}$ for the 4.5 -nm-thick CoSi sample could be due to the contribution from the surface roughness [36]. Based on the definition of $\sigma_{\mathrm{DL}(\mathrm{FL})} \equiv \xi_{\mathrm{DL}(\mathrm{FL})} / \rho_{\mathrm{CoSi}}$, the equivalent spin Hall conductivities of dampinglike and fieldlike SOT, i.e., $\sigma_{\mathrm{DL}}$ and $\sigma_{\mathrm{FL}}$, are plotted in Fig. 3(h). We further fitted the thickness dependence of $\sigma_{\mathrm{DL}(\mathrm{FL})}$ using the equation [37]

$$
\sigma_{\mathrm{DL}(\mathrm{FL})}(t) \equiv \sigma_{\mathrm{DL}(\mathrm{FL})}\left[1-\operatorname{sech}\left(t_{\mathrm{COSi}} / \lambda\right)\right],
$$

and finally, with $\lambda=6 \mathrm{~nm}$, we obtained $\sigma_{\mathrm{DL}}=$ $45(\hbar / e) \Omega^{-1} \mathrm{~cm}^{-1}$ and $\sigma_{\mathrm{FL}}=95(\hbar / e) \Omega^{-1} \mathrm{~cm}^{-1}$.

The temperature dependence of the magnetotransport for single-layer CoSi was systematically studied. The inset of Fig. 4(a) shows the temperature $T$ dependence of the normalized resistivity. We found the temperature coefficient of the resistivity for $\mathrm{CoSi}$ is negative and $\rho_{\mathrm{CoSi}}$ increases by $\sim 10 \%$ as $T$ is swept from 300 to $10 \mathrm{~K}$. We then extract the carrier concentration $n$ and the electron mobility $\mu$ of $\mathrm{CoSi}$ from the slope of the ordinary Hall effect and the longitudinal conductivity. Results are plotted in Fig. 4(a). The temperature dependences of the two quantities are relatively weak. We next investigate the $T$ dependence of the charge-to-spin conversion for a typical $\mathrm{CoSi} / \mathrm{CoFeB}$ bilayer stack with $t_{\mathrm{CoSi}}=8.5 \mathrm{~nm}$ using the harmonic Hall technique. $H_{\text {ext }}$ dependence of $A$ measured at various temperatures is plotted in Fig. 4(b). We found both the dampinglike SOT contribution (exponential decay of $A$ at low field) and ONE contribution (linear slope of $A$ at high field) of $R_{2 \omega}$ reduce with decreasing $T$. At $10 \mathrm{~K}, R_{2 \omega}$ is nearly independent of $H_{\text {ext }}$. Figure 4(c) shows $B$ as a function of $1 / H_{\text {ext }}$ at various temperatures. The slope of $B$ changes sign which indicates a strong temperature dependence of $\xi_{\mathrm{FL}}$. At $10 \mathrm{~K}$, the current-induced Oersted field is sufficient to explain the observed signal, implying that $\xi_{\mathrm{FL}}$ is nearly zero. Figure 4(d) plots the $T$ dependence of $\xi_{\mathrm{DL}}$ and $\xi_{\mathrm{FL}}$. Both $\xi_{\mathrm{DL}}$ and $\xi_{\mathrm{FL}}$ decrease by almost one order of magnitude with decreasing temperature and nearly vanish at $10 \mathrm{~K}$. The $T$ dependences of $\sigma_{\mathrm{DL}}$ and $\sigma_{\mathrm{FL}}$ shown in Fig. 4(e) also exhibit a similar trend. While the $\xi_{\mathrm{FL}}$ of heavy metal/CoFeB/MgO-based heterostructures was known to show such a strong temperature dependence, the $T$ dependence of the dampinglike counterpart in those structures is relatively weak $[38,39]$.

The scaling relationship of $\sigma_{\mathrm{DL}}$ against the transport lifetime $\tau$ is commonly used to separate the intrinsic (independent of $\tau$ ) and the extrinsic skew scattering (proportional to $\tau$ ) contributions [40]. For metals, it is convenient to assume $\sigma_{x x} \sim \tau$. However, for semimetallic $\mathrm{CoSi}$, since $n$ varies with temperature [Fig. 4(a)], we plot $\mu$ instead of $\tau$ in the $x$ axis in Fig. 4(f). For the $y$ axis, we assume $\sigma_{\mathrm{DL}}$ may scale with $n$ due to the thermal excitation [41]. Interestingly, the scaling analyses (whether we divide $\sigma_{\mathrm{DL}}$ by $n$ or not) show a strong temperature dependence of $\sigma_{\mathrm{DL}}$ in $\mathrm{CoSi}$, which neither follows the usual intrinsic nor extrinsic skew scaling $[40,42,43]$, as shown in Fig. 4(f). Thermal-excitation related extra extrinsic scatterings and coupling, such as the local moments [44] of Co ions, and 

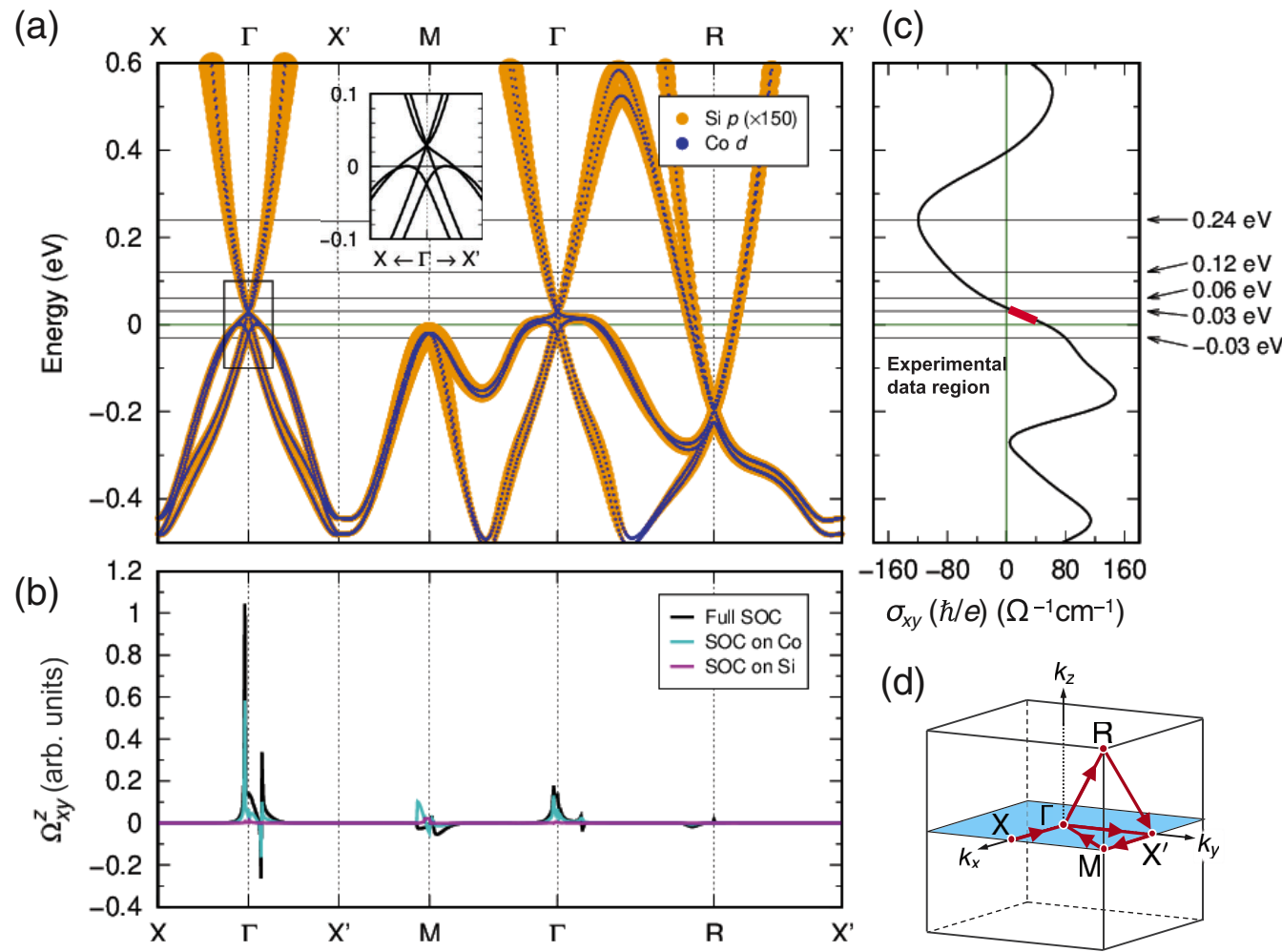

FIG. 5. Band structure and spin Hall conductivity of CoSi. (a) Relativistic band structure of $B 20$-CoSi along high-symmetry $k$ points in the first BZ. Weights of the $\operatorname{Co} d$ (blue) and $\mathrm{Si} p$ (yellow) orbitals are represented by the size of the symbol. The Fermi level is at $E=0 \mathrm{eV}$. Horizontal lines indicate the energy positions relative to the Fermi energy. The inset focuses on the crossing bands of the spin- 1 chiral fermion around $\Gamma$. (b) Spin Berry curvature $\left(\Omega_{x y}^{z}\right)$ contributions along the high-symmetry $k$-point paths on the Fermi level. The results from the full SOC (black) and the SOC on only Co (cyan) or Si (magenta) are shown. (c) Energy dependence of calculated spin Hall conductivity, $\sigma_{x y}$, in the rigid band model. The region of experimental results is also indicated. (d) Illustration of the first BZ showing the $k$ paths. Note that $X$ is at $(0.5,0,0)$ and $X^{\prime}$ is at $(0,0.5,0)$, respectively.

phonons [45], as well as the shift of the Fermi level with its special electronic structures [46], may contribute to the charge-to-spin conversion in $\mathrm{CoSi}$ at elevated temperatures.

\section{FIRST-PRINCIPLES CALCULATIONS}

In order to shed light on the mechanism of the spin-current generation in $\mathrm{CoSi}$ thin films, first-principles calculations based on the density-functional theory (DFT) [47-49] were carried out to evaluate the intrinsic SHC in the B20-type $\mathrm{CoSi}$. The relativistic band structures along high-symmetry $\boldsymbol{k}$ paths are presented in Fig. 5(a). Band crossings with high-fold degeneracy are confirmed at $\Gamma$ (near the Fermi energy) and $R$ (at $0.2 \mathrm{eV}$ below the Fermi energy) in the Brillouin zone (BZ) as reported previously [19-21]. These states were identified as the spin-1 chiral fermions and the double Weyl fermions, respectively [18]. The degeneracy of these states, as shown in Fig. 5(a) and its inset, is reduced by the spin-orbit coupling (SOC) and the split bands might be an origin of the enhanced SHC in CoSi. Figure 5(b) (black line) presents the contributions of the spin Berry curvature along the corresponding high-symmetry $\boldsymbol{k}$ paths at the Fermi energy. A remarkable enhancement of the spin Berry curvature arises near the $\Gamma$ point depending on the particular directions, namely, the $\Gamma-X, \Gamma-X^{\prime}$, and $\Gamma-M$ directions. The largest contribution is found in the vicinity of $\Gamma$ in the $\Gamma-X$ line, where $X$ is at $(0.5,0,0)$. In contrast to $\Gamma-X$, both positive and negative contributions can be seen at the $\Gamma-X^{\prime}$ line, where $X^{\prime}$ is at $(0,0.5,0)$. A smaller value of $\Omega_{x y}^{z}$ is obtained in the $\Gamma-M$ line. The calculations obtain a SHC of $\sim 52(\hbar / e) \Omega^{-1} \mathrm{~cm}^{-1}$ on the Fermi level. Figure 5(c) shows the energy dependence of the SHC within the rigid band model, indicating the value and sign of the SHC depend on the Fermi energy. The maximum amplitudes of the SHC are found to be $\sim 147$ and $\sim 119(\hbar / e) \Omega^{-1} \mathrm{~cm}^{-1}$ when the Fermi energy shifts down to -0.16 and up to $0.24 \mathrm{eV}$, respectively. For the former (at -0.16 $\mathrm{eV}$ ), it is confirmed that the spin Berry curvature is enhanced near the band crossing of the double Weyl point at $R$ in the BZ. Therefore, controlling the Fermi energy by doping with other elements could be an effective way to achieve a larger SHC in this material.

Interestingly, SHC vanishes at the spin-1 band crossing and changes its sign above and below the topological node, whereas a rather peaklike structure of SHC was observed around the double Weyl point [Fig. 5(c)]. The distinct oddfunction-like energy dependence of SHC for the spin-1 chiral fermion clearly distinguishes itself from others (e.g., massive Dirac fermion [50], inversion asymmetric Weyl fermion [12], magnetic Weyl fermion [51], and Dirac fermion from the topological surface states [46]) where the Fermi-energy 
(a) $0.24 \mathrm{eV}$

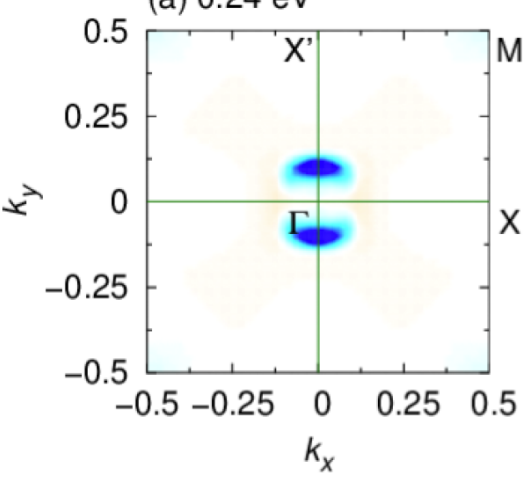

(d) $0.03 \mathrm{eV}$

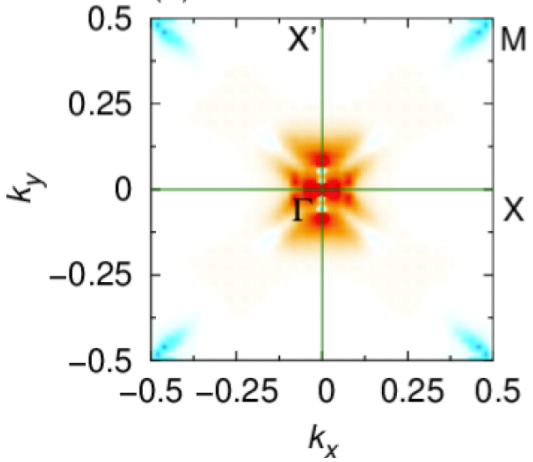

(b) $0.12 \mathrm{eV}$

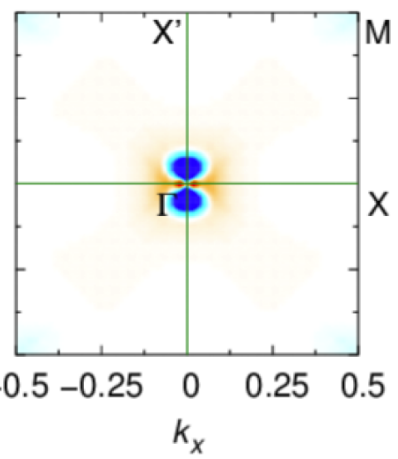

(e) $0.00 \mathrm{eV}$

(e) $0.00 \mathrm{eV}$

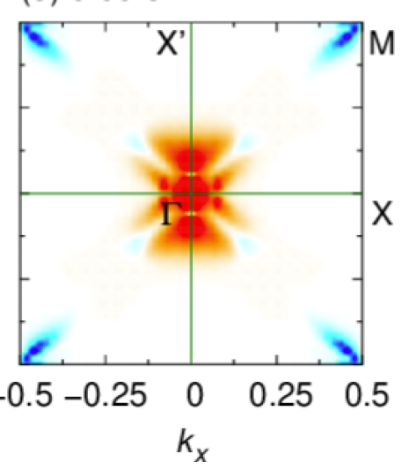

(c) $0.06 \mathrm{eV}$

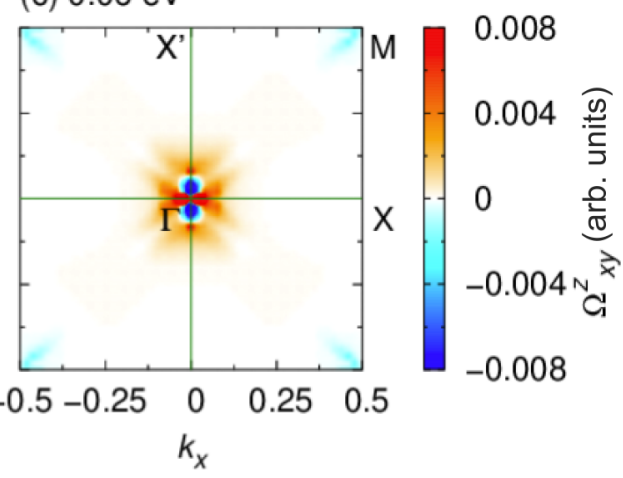

(f) $-0.03 \mathrm{eV}$

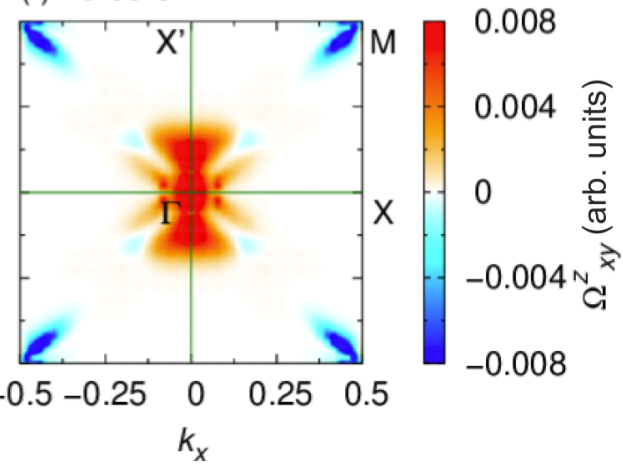

FIG. 6. Spin Berry curvature contributions projected onto the $k_{x} k_{y}$ plane at $k_{z}=0$ in the BZ. (a) Energy slice at 0.24 , (b) 0.12 , (c) 0.06 , (d) 0.03 , (e) 0.00 (Fermi level), and (f) $-0.03 \mathrm{eV}$, respectively [see also Fig. 5(a) and 5(b)]. The color bar indicates positive (red) and negative (blue) contributions of $\Omega_{x y}^{z}$. The $k_{x} k_{y}$ plane corresponds to filled area (blue) in Fig. 5(d).

dependence of the (anomalous and spin) Hall conductivities are rather evenlike.

To provide more insights into this unique character, we show the contributions of $\Omega_{x y}^{z}$ on the slices of BZ $\left(k_{x} k_{y}\right.$ plane at $\left.k_{z}=0\right)$ at varying energies of $0.24,0.12,0.06,0.03,0$, and $-0.03 \mathrm{eV}$ in Figs. 6(a)-6(f), respectively. At the spin-1 chiral band-crossing point $[0.03 \mathrm{eV}$, Fig. 6(d)], the positive contribution of $\Omega_{x y}^{z}$ is found around the $\Gamma$ point with twofold symmetry. When the energy shifts upward to $0.06 \mathrm{eV}$ [Fig. 6(c)], the negative $\Omega_{x y}^{z}$ appears along the $\Gamma-X^{\prime}$ line. By further increasing the energy to 0.12 [Fig. 6(b)] and to 0.24 $\mathrm{eV}$ [Fig. 6(a)], the negative contribution of $\Omega_{x y}^{z}$ is increased and eventually, the value of SHC reaches the minimum value, $-119(\hbar / e) \Omega^{-1} \mathrm{~cm}^{-1}$, at $0.24 \mathrm{eV}$. On the other hand, the $\Omega_{x y}^{z}$ values increase with decreasing energy to the Fermi level $(0 \mathrm{eV})$ and to $-0.03 \mathrm{eV}$ [Figs. 6(e) and 6(f)], resulting in the positive SHC below the spin-1 chiral bands.

It is found that the value of the spin Berry curvature can be understood in terms of the $d$ - $p$ orbital hybridization between the nearest $\mathrm{Co}$ and $\mathrm{Si}$ atoms through the electric dipole transition from occupied $d$ states to unoccupied $p$ states. In Fig. 5(a), orbital weights of Co $d$ states are dominant below the spin- 1 chiral fermions and these orbitals hybridize with a small component of the Si $p$ orbital. The hybridization of Co $d$ and Si $p$ orbitals plays an important role in determining the $\mathrm{SHC}$ in the CoSi. According to the selection rule for the electric dipole transitions [52], the transitions from the occupied $d$ state of $|\boldsymbol{k} l m n\rangle$ with angular quantum number $l=2$ to the unoccupied $p$ state of $\left\langle\boldsymbol{k} l^{\prime} m^{\prime} n^{\prime}\right|$ with $l^{\prime}=1$ that satisfy mag- netic quantum number $m=m^{\prime}-1\left(m=m^{\prime}+1\right)$ may give a positive (negative) contribution to the $\mathrm{SHC}$ ( $n$ is a band index). Below the spin-1 chiral fermions, our calculations clarified that the positive value of SHC originates from the transitions satisfying $l=l^{\prime}+1$ and $m=m^{\prime}-1$, specifically, from the $d_{-1}$ state $(l=2, m=-1)$ to the $p_{0}$ state $\left(l^{\prime}=1, m^{\prime}=0\right)$ and from the $d_{-2}$ state to the $p_{-1}$ state along the $\Gamma-X^{\prime}$ line, and the $d_{0}$ state to the $p_{1}$ state along the $\Gamma-X$ line, respectively. Note the transitions $d_{1} \rightarrow p_{0}$ and $d_{2} \rightarrow p_{1}$ that give negative contributions are also confirmed around the Fermi level. These transitions cancel a part of the positive contributions; thus the resulting value of SHC in CoSi is relatively small at the Fermi level. In the higher-energy region $(E>0.03 \mathrm{eV})$, the transitions $d_{0} \rightarrow p_{-1}$ were found to give negative SHC. We mention that above and below the double Weyl point, the positive $\Omega_{x y}^{z}$ is attributed to $d_{-1} \rightarrow p_{0}$ transitions around the $R$ point of the $\Gamma-R$ line.

We further emphasize that the small Si component hybridizing with the Co $d$ states is an essential ingredient for the SHC in CoSi. Further DFT calculations were carried out for $B 20-C o S i$ where the SOC of either Co or Si is turned off. Since the SOC in Si is very weak, the calculations with SOC only for Si obtain a tiny value of the $\mathrm{SHC},-1(\hbar / e) \Omega^{-1} \mathrm{~cm}^{-1}$ [magenta line in Fig. 5(b)]. In contrast, the inclusion of the SOC only for Co raises the SHC up to $\sim 38(\hbar / e) \Omega^{-1} 1 \mathrm{~cm}^{-1}$ [cyan line in Fig. 5(b)]; however, this value is much smaller than the SHC of CoSi, where the SOC of both atoms is turned on. Particularly, the peak of spin Berry curvature along the $\Gamma-X$ line is doubled for the full SOC case as shown in 
Fig. 5(b). Thus, the bonding of Si $p$ states with Co $d$ states plays a significant role in the SHC despite the "small" $(\sim 1 \%)$ amount of the Si $p$ character in the hybridized band structures.

As a final remark, the vanishing of SHC contribution at the spin-1 chiral crossing near the Fermi level results in the relatively small $\xi_{\mathrm{DL}}$ observed in this study. The SHC exhibits an odd-function-like energy dependence for the spin- 1 chiral fermion. The sign of SHC changes above and below the topological node and the amplitude of SHC is close to zero at the spin-1 chiral crossing. Since the spin-1 chiral crossing locates very near the Fermi level, the $\xi_{\text {DL }}$ proportional to SHC is small. The local derivative in energy of SHC $\partial \sigma_{x y} / \partial E$ is, however, robust near the topological node and may be exploited for thermal spin-current generation via the spin Nernst effect $[53,54]$. Furthermore, it would be interesting to investigate the isostructural $B 20 \mathrm{PtAl}$ [55] for which dramatic enhancement of SOC is expected to boost $\partial \sigma_{x y} / \partial E$ at the crossing and peaks of $\sigma_{x y}$ slightly away from the crossing.

\section{CONCLUSIONS}

In summary, the spin-1 chiral CoSi semimetal thin films and heterostructures were fabricated for quantifying the SOTs. The CoSi films grown on sapphire $c$-plane substrates showed a $B 20$ crystal structure in the polycrystalline phase with flat surface morphology. The spin-current generation in the CoSi films via SHE was investigated by SMR and harmonic Hall measurements. The spin Hall efficiency of the CoSi films was evaluated to be $\sim 3 \%$ at room temperature and decreased with decreasing temperature. First-principles calculations indicate that the hybridization between $d-p$ orbitals results in a large enhancement of spin Berry curvature, which mainly contributes to the SHC in the CoSi. The unique antisymmetriclike energy dependence of $\mathrm{SHC}$ highlights the critical role of Fermi level tuning for harnessing all the benefits of exotic spin-1 chiral fermions for spin-current generation driven by either an electric field or a thermal gradient.

\section{ACKNOWLEDGMENTS}

K. Tang acknowledges National Institute for Materials Science for the provision of a NIMS Junior Research Assistantship. We thank Kohji Nakamura at Mie University and Guanxiong Qu at University of Tokyo for fruitful discussions. This work was partially supported by the KAKENHI (Grants No. JP20K04569, No. JP20H02190, No. JP20H00299, No. JP16H06332, and No. JP20K15156) from the Japan Society for the Promotion of Science (JSPS), the Inter-University Cooperative Research Program of the Institute for Materials Research, Tohoku University (Grant No. 20K0058).

K. Tang, Y.L., and K.N. contributed equally to this work.

\section{APPENDIX: MATERIALS AND METHODS}

\section{Film deposition, characterizations, and device fabrication}

The CoSi thin films were deposited using magnetron sputtering at the substrate temperature of $550{ }^{\circ} \mathrm{C}$ in a highvacuum sputter chamber. The base and deposition pressures were $2 \times 10^{-6}$ and $0.7 \mathrm{~Pa}$ (Ar gas), respectively. The sputtering power of $\mathrm{CoSi}$ was set at $20 \mathrm{~W}$ generated by a direct current (dc) source for a $76.2 \mathrm{~mm}$ diameter $\mathrm{Co}_{50} \mathrm{Si}_{50}$ target. The crystal structure was characterized using the out-of-plane XRD measurement with $\mathrm{Cu} K \alpha$ radiation $(\lambda=$ $0.15418 \mathrm{~nm}$ ). The surface structure and morphology were evaluated using RHEED and AFM, respectively. The sample with the structure of sapphire(0001) substrate/CoSi $(0-11 \mathrm{~nm}) / \mathrm{CoFeB}(1 \mathrm{~nm}) / \mathrm{MgO}(2 \mathrm{~nm}) / \mathrm{Ta}(1 \mathrm{~nm})$ was postannealed at $350^{\circ} \mathrm{C}$ for $1 \mathrm{~h}$ and was then patterned into Hall bar devices (width: $10 \mu \mathrm{m}$, length: $25 \mu \mathrm{m}$ ) by conventional UV lithography and Ar ion milling. Ta(5)/ $\mathrm{Au}(150 \mathrm{~nm})$ layers were then deposited on the Hall bars as electrodes using a lift-off process.

\section{SMR and harmonic Hall measurements}

Magnetotransport properties were characterized in a physical properties measurement system (PPMS). For the SMR measurement, a magnetic field of $20 \mathrm{kOe}$ was applied to saturate the magnetization of the FM layer. For the harmonic Hall measurement, a sinusoidal signal of constant amplitude and frequency of $172.1 \mathrm{~Hz}$ was applied by a Keithley 6221 current source meter. The first- and second-harmonic Hall voltages were simultaneously measured by two lock-in amplifiers (nf LI5660).

\section{First-principles calculations}

The first-principles calculations were performed based on the generalized gradient approximation [56] using the fullpotential linearized augmented plane-wave (FLAPW) method [47-49]. The cubic B20-type crystal structure of stoichiometric CoSi was modeled by using the experimental lattice constant, in which all the atomic positions were fully relaxed by the force calculations [57]. Muffin-tin (MT) radii of 2.40 and $1.80 a_{\mathrm{B}}$ were employed for Co and $\mathrm{Si}$, respectively. The LAPW basis for the wave function in the interstitial region has a cutoff of $|\boldsymbol{k}+\boldsymbol{G}| \leqslant 4.5 a_{\mathrm{B}}^{-1}$, and the angular momentum expansion inside the MT sphere is truncated at $\ell=8$ for the $\mathrm{Co}$ and at 6 for the O. The SOC was incorporated by the second variational method [58]. The intrinsic SHC was evaluated by means of the Kubo formula in the static limit $(\omega=0)[59,60]$,

$$
\sigma_{x y}=\frac{e}{\hbar} \sum_{k} \sum_{n} f_{k n} \Omega^{z}(\boldsymbol{k}),
$$

where the spin Berry curvature $\Omega^{z}(\boldsymbol{k})$ is given by

$$
\Omega^{z}(\boldsymbol{k})=\sum_{n^{\prime}} \frac{2 \operatorname{Im}\left\langle\boldsymbol{k} n\left|\hat{j}_{x}\right| \boldsymbol{k} n^{\prime}\right\rangle\left\langle\boldsymbol{k} n^{\prime}\left|\hat{v}_{y}\right| \boldsymbol{k} n\right\rangle}{\left(\varepsilon_{\boldsymbol{k} n}-\varepsilon_{\boldsymbol{k} n^{\prime}}\right)^{2}} .
$$

Here, $f_{k n}$ is the Fermi distribution function for the $n$th band at $\boldsymbol{k}, \hat{j}_{x}$ and $\hat{v}_{y}$ are the spin and charge current operators, and $\varepsilon_{\boldsymbol{k} n}$ and $\varepsilon_{\boldsymbol{k} n^{\prime}}$ are the calculated eigenvalues of the occupied and unoccupied states, $|\boldsymbol{k} n\rangle$ and $\left|\boldsymbol{k} n^{\prime}\right\rangle$, respectively. With a $55 \times$ $55 \times 55$ special $\boldsymbol{k}$-point mesh in the BZ, the SHC was found to sufficiently suppress numerical fluctuations. 
[1] S. Fukami, T. Anekawa, C. Zhang, and H. Ohno, A spin-orbit torque switching scheme with collinear magnetic easy axis and current configuration, Nat. Nanotechnol. 11, 621 (2016).

[2] G. Yu, Two-terminal MRAM with a spin, Nat. Electron. 1, 496 (2018).

[3] T. Seki, Y. Hasegawa, S. Mitani, S. Takahashi, H. Imamura, S. Maekawa, J. Nitta, and K. Takanashi, Giant spin Hall effect in perpendicularly spin-polarized FePt/Au devices, Nat. Mater. 7, 125 (2008).

[4] M. Althammer, S. Meyer, H. Nakayama, M. Schreier, S. Altmannshofer, M. Weiler, H. Huebl, S. Geprägs, M. Opel, R. Gross, D. Meier, C. Klewe, T. Kuschel, J.-M. Schmalhorst, G. Reiss, L. Shen, A. Gupta, Y.-T. Chen, G. E. W. Bauer, E. Saitoh, and S. T. B. Goennenwein, Quantitative study of the spin Hall magnetoresistance in ferromagnetic insulator/normal metal hybrids, Phys. Rev. B 87, 224401 (2013).

[5] L. Liu, C.-F. Pai, Y. Li, H. W. Tseng, D. C. Ralph, and R. A. Buhrman, Spin-torque switching with the giant spin Hall effect of tantalum, Science 336, 555 (2012).

[6] C.-F. Pai, L. Liu, Y. Li, H. W. Tseng, D. C. Ralph, and R. A. Buhrman, Spin transfer torque devices utilizing the giant spin Hall effect of tungsten, Appl. Phys. Lett. 101, 122404 (2012).

[7] A. R. Mellnik, J. S. Lee, A. Richardella, J. L. Grab, P. J. Mintun, M. H. Fischer, A. Vaezi, A. Manchon, E.-A. Kim, N. Samarth, and D. C. Ralph, Spin-transfer torque generated by a topological insulator, Nature 511, 449 (2014).

[8] M. Jamali, J. S. Lee, J. S. Jeong, F. Mahfouzi, Y. Lv, Z. Zhao, B. K. Nikolić, K. A. Mkhoyan, N. Samarth, and J.-P. Wang, Giant spin pumping and inverse spin Hall effect in the presence of surface and bulk spin-orbit coupling of topological insulator $\mathrm{Bi}_{2} \mathrm{Se}_{3}$, Nano Lett. 15, 7126 (2015).

[9] Y. Wang, D. Zhu, Y. Wu, Y. Yang, J. Yu, R. Ramaswamy, R. Mishra, S. Shi, M. Elyasi, K.-L. Teo, Y. Wu, and H. Yang, Room temperature magnetization switching in topological insulator-ferromagnet heterostructures by spin-orbit torques, Nat. Commun. 8, 1364 (2017).

[10] M. DC, R. Grassi, J.-Y. Chen, M. Jamali, D. R. Hickey, D. Zhang, Z. Zhao, H. Li, P. Quarterman, Y. Lv, M. Li, A. Manchon, K. A. Mkhoyan, T. Low, and J.-P. Wang, Room-temperature high spin-orbit torque due to quantum confinement in sputtered $\left.\mathrm{Bi}_{x} \mathrm{Se}_{1-x}\right)$ films, Nat. Mater. 17, 800 (2018).

[11] B. Yan and C. Felser, Topological materials: Weyl semimetals, Annu. Rev. Condens. Matter Phys. 8, 337 (2017).

[12] Y. Sun, Y. Zhang, C. Felser, and B. Yan, Strong Intrinsic Spin Hall Effect in the TaAs Family of Weyl Semimetals, Phys. Rev. Lett. 117, 146403 (2016).

[13] D. MacNeill, G. M. Stiehl, M. H. D. Guimaraes, R. A. Buhrman, J. Park, and D. C. Ralph, Control of spin-orbit

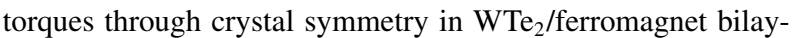
ers, Nat. Phys. 13, 300 (2016).

[14] S. Shi, S. Liang, Z. Zhu, K. Cai, S. D. Pollard, Y. Wang, J. Wang, Q. Wang, P. He, J. Yu, G. Eda, G. Liang, and H. Yang, All-electric magnetization switching and DzyaloshinskiiMoriya interaction in $\mathrm{WTe}_{2}$ /ferromagnet heterostructures, Nat. Nanotechnol. 14, 10 (2019).

[15] K. Tang, Z. Wen, Y.-C. Lau, H. Sukegawa, T. Seki, and S. Mitani, Magnetization switching induced by spin-orbit torque from $\mathrm{Co}_{2} \mathrm{MnGa}$ magnetic Weyl semimetal thin films, Appl. Phys. Lett. 118, 062402 (2021).
[16] L. Leiva, S. Granville, Y. Zhang, S. Dushenko, E. Shigematsu, T. Shinjo, R. Ohshima, Y. Ando, and M. Shiraishi, Giant spin Hall angle in the Heusler alloy Weyl ferromagnet $\mathrm{Co}_{2} \mathrm{MnGa}$, Phys. Rev. B 103, L041114 (2021).

[17] B. Bradlyn, J. Cano, Z. Wang, M. G. Vergniory, C. Felser, R. J. Cava, and B. A. Bernevig, Beyond Dirac and Weyl fermions: Unconventional quasiparticles in conventional crystals, Science 353, aaf5037 (2016).

[18] P. Tang, Q. Zhou, and S.-C. Zhang, Multiple Types of Topological Fermions in Transition Metal Silicides, Phys. Rev. Lett. 119 , 206402 (2017).

[19] D. Takane, Z. Wang, S. Souma, K. Nakayama, T. Nakamura, H. Oinuma, Y. Nakata, H. Iwasawa, C. Cacho, T. Kim, K. Horiba, H. Kumigashira, T. Takahashi, Y. Ando, and T. Sato, Observation of Chiral Fermions with a Large Topological Charge and Associated Fermi-Arc Surface States in CoSi, Phys. Rev. Lett. 122, 076402 (2019).

[20] D. S. Sanchez, I. Belopolski, T. A. Cochran, X. Xu, J.-X. Yin, G. Chang, W. Xie, K. Manna, V. Süß, C.-Y. Huang, N. Alidoust, D. Multer, S. S. Zhang, N. Shumiya, X. Wang, G.-Q. Wang, T.-R. Chang, C. Felser, S.-Y. Xu, S. Jia et al., Topological chiral crystals with helicoid-arc quantum states, Nature 567, 500 (2019).

[21] Z. Rao, H. Li, T. Zhang, S. Tian, C. Li, B. Fu, C. Tang, L. Wang, Z. Li, W. Fan, J. Li, Y. Huang, Z. Liu, Y. Long, C. Fang, H. Weng, Y. Shi, H. Lei, Y. Sun, T. Qian et al., Observation of unconventional chiral fermions with long Fermi arcs in CoSi, Nature 567, 496 (2019).

[22] H. Wang, C. Du, P. C. Hammel, and F. Yang, Spin current and inverse spin hall effect in ferromagnetic metals probed by $\mathrm{Y}_{3} \mathrm{Fe}_{5} \mathrm{O}_{12}$-based spin pumping, Appl. Phys. Lett. 104, 202405 (2014).

[23] K. Ando and E. Saitoh, Observation of the inverse spin Hall effect in silicon, Nat. Commun. 3, 629 (2012).

[24] H. Nakayama, M. Althammer, Y.-T. Chen, K. Uchida, Y. Kajiwara, D. Kikuchi, T. Ohtani, S. Geprägs, M. Opel, S. Takahashi, R. Gross, G. E. W. Bauer, S. T. B. Goennenwein, and E. Saitoh, Spin Hall Magnetoresistance Induced by a Nonequilibrium Proximity Effect, Phys. Rev. Lett. 110, 206601 (2013).

[25] J. Kim, P. Sheng, S. Takahashi, S. Mitani, and M. Hayashi, Spin Hall Magnetoresistance in Metallic Bilayers, Phys. Rev. Lett. 116, 097201 (2016).

[26] J. Liu, T. Ohkubo, S. Mitani, K. Hono, and M. Hayashi, Correlation between the spin Hall angle and the structural phases of early $5 d$ transition metals, Appl. Phys. Lett. 107, 232408 (2015).

[27] Y.-T. Chen, S. Takahashi, H. Nakayama, M. Althammer, S. T. B. Goennenwein, E. Saitoh, and G. E. W. Bauer, Theory of spin Hall magnetoresistance, Phys. Rev. B 87, 144411 (2013).

[28] X. Xu, X. Wang, T. A. Cochran, D. S. Sanchez, G. Chang, I. Belopolski, G. Wang, Y. Liu, H.-J. Tien, X. Gui, W. Xie, M. Z. Hasan, T.-R. Chang, and S. Jia, Crystal growth and quantum oscillations in the topological chiral semimetal CoSi, Phys. Rev. B 100, 045104 (2019).

[29] C. O. Avci, K. Garello, M. Gabureac, A. Ghosh, A. Fuhrer, S. F. Alvarado, and P. Gambardella, Interplay of spin-orbit torque and thermoelectric effects in ferromagnet/normal-metal bilayers, Phys. Rev. B 90, 224427 (2014).

[30] J. Kim, J. Sinha, M. Hayashi, M. Yamanouchi, S. Fukami, T. Suzuki, S. Mitani, and H. Ohno, Layer thickness dependence 
of the current-induced effective field vector in $\mathrm{Ta}|\mathrm{CoFeB}| \mathrm{MgO}$, Nat. Mater. 12, 240 (2013).

[31] K. Garello, I. M. Miron, C. O. Avci, F. Freimuth, Y. Mokrousov, S. Blügel, S. Auffret, O. Boulle, G. Gaudin, and P. Gambardella, Symmetry and magnitude of spin-orbit torques in ferromagnetic heterostructures, Nat. Nanotechnol. 8, 587 (2013).

[32] Z. Wen, J. Kim, H. Sukegawa, M. Hayashi, and S. Mitani, Spinorbit torque in $\mathrm{Cr} / \mathrm{CoFe} \mathrm{Al} / \mathrm{MgO}$ and $\mathrm{Ru} / \mathrm{CoFeAl} / \mathrm{MgO}$ epitaxial magnetic heterostructures, AIP Adv. 6, 056307 (2016).

[33] Y.-C. Lau, H. Lee, G. Qu, K. Nakamura, and M. Hayashi, Spin Hall effect from hybridized $3 d-4 p$ orbitals, Phys. Rev. B 99, 064410 (2019).

[34] Y.-C. Lau and M. Hayashi, Spin torque efficiency of Ta, W, and $\mathrm{Pt}$ in metallic bilayers evaluated by harmonic Hall and spin Hall magnetoresistance measurements, Jpn. J. Appl. Phys. 56, 0802B5 (2017).

[35] L. Liu, O. J. Lee, T. J. Gudmundsen, D. C. Ralph, and R. A. Buhrman, Current-Induced Switching of Perpendicularly Magnetized Magnetic Layers Using Spin Torque from the Spin Hall Effect, Phys. Rev. Lett. 109, 096602 (2012).

[36] L. Zhou, V. L. Grigoryan, S. Maekawa, X. Wang, and J. Xiao, Spin Hall effect by surface roughness, Phys. Rev. B 91, 045407 (2015).

[37] L. Liu, T. Moriyama, D. C. Ralph, and R. A. Buhrman, SpinTorque Ferromagnetic Resonance Induced by the Spin Hall Effect, Phys. Rev. Lett. 106, 036601 (2011).

[38] J. Kim, J. Sinha, S. Mitani, M. Hayashi, S. Takahashi, S. Maekawa, M. Yamanouchi, and H. Ohno, Anomalous temperature dependence of current-induced torques in $\mathrm{CoFeB} / \mathrm{MgO}$ heterostructures with Ta-based underlayers, Phys. Rev. B 89, 174424 (2014).

[39] Y. Ou, C.-F. Pai, S. Shi, D. C. Ralph, and R. A. Buhrman, Origin of fieldlike spin-orbit torques in heavy metal/ferromagnet/oxide thin film heterostructures, Phys. Rev. B 94, 140414(R) (2016).

[40] J. Sinova, S. O. Valenzuela, J. Wunderlich, C. H. Back, and T. Jungwirth, Spin Hall effects, Rev. Mod. Phys. 87, 1213 (2015).

[41] Z. Chi, Y.-C. Lau, X. Xu, T. Ohkubo, K. Hono, and M. Hayashi, The spin Hall effect of Bi-Sb alloys driven by thermally excited Dirac-like electrons, Sci. Adv. 6, eaay2324 (2020).

[42] Y. Niimi, M. Morota, D. H. Wei, C. Deranlot, M. Basletic, A. Hamzic, A. Fert, and Y. Otani, Extrinsic Spin Hall Effect Induced by Iridium Impurities in Copper, Phys. Rev. Lett. 106, 126601 (2011).

[43] E. Sagasta, Y. Omori, M. Isasa, M. Gradhand, L. E. Hueso, Y. Niimi, Y. C. Otani, and F. Casanova, Tuning the spin Hall effect of Pt from the moderately dirty to the superclean regime, Phys. Rev. B 94, 060412(R) (2016).

[44] S. Okamoto, T. Egami, and N. Nagaosa, Critical Spin Fluctuation Mechanism for the Spin Hall Effect, Phys. Rev. Lett. 123, 196603 (2019).

[45] C. Gorini, U. Eckern, and R. Raimondi, Spin Hall Effects Due to Phonon Skew Scattering, Phys. Rev. Lett. 115, 076602 (2015).

[46] K. Kondou, R. Yoshimi, A. Tsukazaki, Y. Fukuma, J. Matsuno, K. S. Takahashi, M. Kawasaki, Y. Tokura, and Y. Otani, Fermilevel-dependent charge-to-spin current conversion by Dirac surface states of topological insulators, Nat. Phys. 12, 1027 (2016).

[47] E. Wimmer, H. Krakauer, M. Weinert, and A. J. Freeman, Full-potential self-consistent linearized-augmented-plane-wave method for calculating the electronic structure of molecules and surfaces: $\mathrm{O}_{2}$ molecule, Phys. Rev. B 24, 864 (1981).

[48] M. Weinert, E. Wimmer, and A. J. Freeman, Total-energy allelectron density functional method for bulk solids and surfaces, Phys. Rev. B 26, 4571 (1982).

[49] K. Nakamura, T. Ito, A. J. Freeman, L. Zhong, and J. Fernandez-de-Castro, Enhancement of magnetocrystalline anisotropy in ferromagnetic $\mathrm{Fe}$ films by intra-atomic noncollinear magnetism, Phys. Rev. B 67, 014420 (2003).

[50] L. Ye, M. Kang, J. Liu, F. von Cube, C. R. Wicker, T. Suzuki, C. Jozwiak, A. Bostwick, E. Rotenberg, D. C. Bell, L. Fu, R. Comin, and J. G. Checkelsky, Massive Dirac fermions in a ferromagnetic kagome metal, Nature 555, 7698 (2018).

[51] E. Liu, Y. Sun, N. Kumar, L. Muechler, A. Sun, L. Jiao, S.-Y. Yang, D. Liu, A. Liang, Q. Xu, J. Kroder, V. Süß, H. Borrmann, C. Shekhar, Z. Wang, C. Xi, W. Wang, W. Schnelle, S. Wirth, Y. Chen et al., Giant anomalous Hall effect in a ferromagnetic kagome-lattice semimetal, Nat. Phys. 14, 1125 (2018).

[52] A.-M. Pradipto, T. Akiyama, T. Ito, and K. Nakamura, External electric field driven modification of the anomalous and spin Hall conductivities in Fe thin films on $\mathrm{MgO}(001)$, Phys. Rev. B 97, 024401 (2018).

[53] S. Meyer, Y. T. Chen, S. Wimmer, M. Althammer, T. Wimmer, R. Schlitz, S. Geprags, H. Huebl, D. Kodderitzsch, H. Ebert, G. E. W. Bauer, R. Gross, and S. T. B. Goennenwein, Observation of the spin Nernst effect, Nat. Mater. 16, 97 (2017).

[54] P. Sheng, Y. Sakuraba, Y. C. Lau, S. Takahashi, S. Mitani, and M. Hayashi, The spin Nernst effect in tungsten, Sci. Adv. 3, e1701503 (2017).

[55] N. B. M. Schröter, D. Pei, M. G. Vergniory, Y. Sun, K. Manna, F. de Juan, J. A. Krieger, V. Süss, M. Schmidt, P. Dudin, B. Bradlyn, T. K. Kim, T. Schmitt, C. Cacho, C. Felser, V. N. Strocov, and Y. Chen, Chiral topological semimetal with multifold band crossings and long fermi arcs, Nat. Phys. 15, 759 (2019).

[56] J. P. Perdew, K. Burke, and M. Ernzerhof, Generalized Gradient Approximation Made Simple, Phys. Rev. Lett. 77, 3865 (1996).

[57] R. Yu, D. Singh, and H. Krakauer, All-electron and pseudopotential force calculations using the linearized-augmented-planewave method, Phys. Rev. B 43, 6411 (1991).

[58] C. Li, A. J. Freeman, H. J. F. Jansen, and C. L. Fu, magnetic anisotropy in low-dimensional ferromagnetic systems: Fe monolayers on $\operatorname{Ag}(001), \operatorname{Au}(001)$, and $\mathrm{Pd}(001)$ Substrates, Phys. Rev. B 42, 5433 (1990).

[59] G. Y. Guo, S. Murakami, T.-W. Chen, and N. Nagaosa, Intrinsic Spin Hall Effect in Platinum: First-Principles Calculations, Phys. Rev. Lett. 100, 096401 (2008).

[60] J. Sinova, D. Culcer, Q. Niu, N. A. Sinitsyn, T. Jungwirth, and A. H. MacDonald, Universal Intrinsic Spin Hall Effect, Phys. Rev. Lett. 92, 126603 (2004). 